tion enrolled thus far and to demonstrate the breadth and potential value of the data generated by the new CARRA Registry.

Methods We requested de-identified counts of several fields collected from the case report forms for subjects with SLE. Patients were eligible for enrollment in the new CARRA registry if they were diagnosed with SLE prior to the age of 18 and had either 1) a new diagnosis of SLE or 2) a flare of lupus nephritis within two years prior to the baseline visit. IRB approval was not required for this data request.

Results To date, 184 patients (pts) have been enrolled; 156 $(85 \%)$ are female. There are 46 black pts, 45 Hispanic pts, 47 white pts, 18 Asian pts and 16 pts were $>1$ race. Over half the pts have private health insurance $(n=95,52 \%)$ and 60 pts (33\%) have Medicaid. Autoantibody positivity was prevalent: 175 pts (95\%) were ANA positive, 109 (59\%) dsDNA positive, and 87 (47\%) anti-Smith positive. Positivity for anti-RNP, antiRo, anti-La, and APLs ranged from $15 \%$ to $51 \%$. At the baseline visit, the mean Systemic Lupus Erythematosus Disease Activity Index (SLEDAI, $\mathrm{n}=166$ ) score was $5.5 \pm 6.3$, median $=4$ (range 0-37; IQR 0.25-8). The mean Systemic Lupus International Collaborating Clinic Damage Index (SLICC DI, $n=150$ ) score was 0.4 , median $=0$ (range 0-7). Approximately one quarter of pts $(n=50)$ were being treated for lupus nephritis at the time of the baseline visit. Manifestations of SLE at the baseline visit were varied (table 1) but serologic disease, mucocutaneous disease and active nephritis were the most prevalent. Conclusions Nearly 200 SLE pts have been enrolled in the new CARRA Registry to date. This is a multi-racial cohort with moderate disease activity and varied disease manifestations. Further enrollment will continue to build a robust data source to study disease course and outcomes in a pediatric SLE inception cohort.

Acknowledgements The authors wish to acknowledge the Arthritis Foundation for ongoing financial support of CARRA and the CARRA Registry.

\section{CS-03 HYDROXYCHLOROQUINE IN LUPUS PREGNANCY: A META-ANALYSIS OF INDIVIDUAL PARTICIPANT DATA}

${ }^{1}$ Amanda M Eudy* ${ }^{2}$ Michelle Petri, ${ }^{3}$ Rebecca Fischer-Betz, ${ }^{4}$ Abeer Mokbel, ${ }^{5}$ Cecilia Nalli, ${ }^{5}$ Laura Andreoli, ${ }^{5}$ Angela Tincani, ${ }^{6}$ Yair Molad, ${ }^{7}$ Dafna Gladman, ${ }^{7}$ Murray Urowitz, 'Stephen Balevic, 'Megan EB Clowse. 'Duke University Medical Center; ${ }^{2} J o h n s$ Hopkins University School of Medicine; ${ }^{3}$ University Hospital Duesseldorf; ${ }^{4}$ Cairo University Hospital; ${ }^{5}$ Spedali Civili and University of Brescia; ${ }^{6}$ Tel Aviv University; ${ }^{7}$ University of Toronto

\subsection{6/lupus-2018-Ism.38}

Background Our current knowledge about how to treat lupus in pregnancy derives from small prospective or retrospective cohorts. The goal of this individual participant meta-analysis was to pool data from multiple prospective cohorts to answer the clinical question of whether hydroxychloroquine (HCQ) treatment affects pregnancy outcomes.

Methods The literature was searched for prospective cohorts of pregnancies among women with lupus. HCQ use was defined as use any time during pregnancy. Outcomes of interest included fetal loss, preterm birth, high disease, and preeclampsia. Data from each cohort were collected and analyzed individually. Pooled ORs were calculated by random-effect models in Review Manager. Due to multiple pregnancies per patient, one pregnancy was randomly selected per patient. Primary analysis included only women with first trimester visits (6 cohorts). Subgroup analyses were stratified by a history of nephritis, APS, and disease activity at first clinic visit.

Results The literature was searched for prospective cohorts of pregnancies among women with lupus. HCQ use was defined as use any time during pregnancy. Outcomes of interest included fetal loss, preterm birth, high disease, and preeclampsia. Data from each cohort were collected and analyzed individually. Pooled ORs were calculated by random-effect models in Review Manager. Due to multiple pregnancies per patient, one pregnancy was randomly selected per patient. Primary analysis included only women with first trimester visits (6 cohorts). Subgroup analyses were stratified by a history of nephritis, APS, and disease activity at first clinic visit.

Conclusions Our results suggest that among patients with lupus nephritis, HCQ use may decrease the risk of fetal loss. The heterogeneity of data collection suggests the need for a unified approach to identify larger cohorts of lupus pregnancies.

\section{CS-04 ASSOCIATED FACTORS OF LONG-TERM CARDIAC DYSFUNCTION IN A LONGITUDINAL COHORT OF NEONATAL LUPUS}

${ }^{1}$ Amit Saxena*, ${ }^{1}$ Peter M Izmirly, ${ }^{1}$ Rebecca Bomar, ${ }^{1}$ Shireen Golpanian, ${ }^{2}$ Deborah Friedman, ${ }^{1}$ jill P Buyon. 'NYU School of Medicine, New York, NY, USA; ${ }^{2}$ New York Medical College, Valhalla, NY, USA

\subsection{6/lupus-2018-Ism.39}

Background There are no longitudinal studies regarding the long term cardiac health of children with cardiac manifestations of neonatal lupus (NL). This study was performed to evaluate risk factors for morbidity and provide evidence-based guidance regarding the course of cardiac NL.

Methods Echocardiograms throughout life were evaluated in 240 individuals born with cardiac NL from the Research Registry for Neonatal Lupus: 142 were available from ages 01 years, 174 from ages 1-17 years, and 65>17 years. A composite adverse outcome defined as qualitatively decreased left ventricular (LV) function or concurrent use of cardiac

Abstract CS-03 Table 1 Pooled odds ratios for the association of hydroxychloroquine use and pregnancy outcomes

\begin{tabular}{|c|c|c|c|c|}
\hline & Fetal Loss & Preterm Birth & High Disease Activity & Preeclampsia \\
\hline & OR $(95 \% \mathrm{Cl})$ & OR $(95 \% \mathrm{Cl})$ & OR $(95 \% \mathrm{Cl})$ & OR $(95 \% \mathrm{Cl})$ \\
\hline Overall & $0.50(0.27-0.94)$ & $0.95(0.58-1.55)$ & $0.69(0.35-1.39)$ & $1.19(0.62-2.30)$ \\
\hline Lupus Nephritis History & $0.24(0.07-0.83)$ & $0.81(0.35-1.89)$ & $0.47(0.21-1.09)$ & $0.70(0.24-2.03)$ \\
\hline No Lupus Nephritis History & $0.70(0.33-1.46)$ & $1.04(0.57-1.89)$ & $0.98(0.45-2.17)$ & $1.36(0.58-3.16)$ \\
\hline APS & $0.39(0.10-1.47)$ & $0.82(0.23-2.96)$ & $1.30(0.16-10.48)$ & $0.55(0.12-2.45)$ \\
\hline No APS & $0.61(0.31-1.20)$ & $0.96(0.56-1.64)$ & $0.70(0.40-1.22)$ & $1.28(0.58-2.84)$ \\
\hline High Disease Activity at $1^{\text {st }}$ Visit & $0.61(0.13-2.89)$ & $1.53(0.42-5.62)$ & - & $0.93(0.12-7.14)$ \\
\hline No High Disease Activity at $1^{\text {st }}$ Visit & $0.46(0.21-1.02)$ & $0.81(0.45-1.44)$ & $0.73(0.29-1.87)$ & $1.07(0.50-2.31)$ \\
\hline
\end{tabular}

